\title{
CORRIGENDUM
}

\section{Design of a family-based lifestyle intervention for youth with type 2 diabetes: the TODAY study}

\author{
The TODAY Study Group \\ International Journal of Obesity (2010) 34, 946; doi:10.1038/ijo.2009.247
}

Correction to: International Journal of Obesity (2010) 34, 217-226; doi:10.1038/ijo.2009.195; published online 13 October 2010

Since the publication of the above paper, the authors have noticed that there are inadvertent omissions of key staff and minor misspellings of names in the listing of the TODAY Study Group members in Appendix A. The complete list is reproduced below.

\section{Appendix}

TODAY study group: The following individuals and institutions constitute the TODAY Study Group (*indicates principal investigator or director):

CLINICAL CENTERS-Baylor College of Medicine: M Haymond*, B Anderson, C Bush, S Gunn, H Holden, M Jones, K Hwu, S McGirk, S McKay, S Thamotharan, B Schreiner, M Zarate; Case Western Reserve University: L Cuttler*, E Abrams, T Casey, W Dahms, D Drotar, S Huestis, C Ievers-Landis, P McGuigan, S Sundararajan; Childrens Hospital Los Angeles: M Geffner*, N Chang, D Dreimane, M Halvorson, S Hernandez, F Kaufman (Study Chair), V Mansilla, R Ortiz, A Ward, K Wexler, P Yasuda; Children's Hospital of Philadelphia: L Levitt Katz*, R Berkowitz, S Boyd, C Carchidi, B Johnson, J Kaplan, C Keating, S Kneeshaw-Price, C Lassiter, T Lipman, S Magge, G McGinley, B Schwartzman, S Willi; Children's Hospital of Pittsburgh: S Arslanian*, F Bacha, S Foster, B Galvin, T Hannon, A Kriska, I Libman, $\mathrm{M}$ Marcus, $\mathrm{K}$ Porter, $\mathrm{T}$ Songer, $\mathrm{E}$ Venditti; Columbia University Medical Center: R Goland*, R Cain, I Fennoy, D Gallagher, P Kringas, N Leibel, R Motaghedi, D Ng, M Ovalles, M Pellizzari, R Rapaport, K Robbins, D Seidman, L Siegel-Czarkowski, P Speiser; Joslin Diabetes Center: L Laffel*, A Goebel-Fabbri, M Hall, L Higgins, M Malloy, K Milaszewski, L Orkin, A Rodriguez-Ventura; Massachusetts General Hospital: D Nathan*, L Bissett, K Blumenthal, L Delahanty, V Goldman, A Goseco, M Larkin, L Levitsky, R McEachern, K Milaszewski, D Norman, B Nwosu, S Park-Bennett, D Richards, N Sherry, B Steiner; Saint Louis
University: S Tollefsen*, S Carnes, D Dempsher, D Flomo, A Floreen, V Kociela, T Whelan, B Wolff; State University of New York Upstate Medical University: R Weinstock*, D Bowerman, J Bulger, K Duncan, R Franklin, J Hartsig, R Izquierdo, J Kanaley, J Kearns, S Meyer, R Saletsky, P Trief; University of Colorado Health Sciences Center: P Zeitler* (Steering Committee Chair), N Abramson, A Bradhurst, N Celona-Jacobs, J Glazner, J Higgins, F Hoe, G Klingensmith, $\mathrm{K}$ Nadeau, $\mathrm{H}$ Strike, $\mathrm{T}$ Witten; University of Oklahoma Health Sciences Center: K Copeland* (Steering Committee Vice-Chair), R Brown, J Chadwick, L Chalmers, C Macha, A Nordyke, T Poulsen, L Pratt, J Preske, J Schanuel, J Smith, S Sternlof, R Swisher; University of Texas Health Science Center at San Antonio: D Hale*, N Amodei, R Barajas, C Cody, S Haffner, J Hernandez, C Ibarra, J Lynch, E Morales, S Rivera, G Rupert, A Wauters; Washington University School of Medicine: N White*, A Arbeláez, J Jones, T Jones, M Sadler, M Tanner, A Timpson, R Welch; Yale University: S Caprio*, M Grey, C Guandalini, S Lavietes, M Mignosa, P Rose, A Syme, W Tamborlane.

COORDINATING CENTER-George Washington University Biostatistics Center: K Hirst*, L Coombs, S Edelstein, N Grover, C Long, L Pyle.

PROJECT OFFICE - National Institute of Diabetes and Digestive and Kidney Diseases: B Linder*.

CENTRAL UNITS - Central Blood Laboratory (Northwest Lipid Research Laboratories, University of Washington): S Marcovina*, J Chmielewski, M Ramirez, G Strylewicz; DEXA Reading Center (University of California at San Francisco): J Shepherd*, B Fan, L Marquez, M Sherman, J Wang; Diet Assessment Center (University of South Carolina): E Mayer-Davis*, Y Liu, M Nichols; Lifestyle Program Core (Washington University): D Wilfley*, D Aldrich-Rasche, K Franklin, G Leibach, C Massmann, M Mills, D O'Brien, J Patterson, T Tibbs, D Van Buren.

OTHER - Centers for Disease Control: P Zhang; Hospital for Sick Children, Toronto: M Palmert; State University of New York at Buffalo: L Epstein; University of Florida: J Silverstein.

The authors would like to apologize for the mistake. 